\title{
Clinical implications of quantitative real-time RT-PCR analysis of hTERT gene expression in human gliomas
}

\author{
A Tchirkov*,I,2, C Rolhion 1,3, J-L Kémény ${ }^{4}$, B Irthum ${ }^{5}$, S Puget ${ }^{5}$, T Khalii ${ }^{5}$, O Chinot $^{6}$, F Kwiatkowski ${ }^{7}$, \\ B Périssel ${ }^{2}, \mathbf{P}$ Vago $^{2}$ and $\mathbf{P}$ Verrelle ${ }^{1,3}$
}

'Département de Radiothérapie, Centre Jean Perrin, 63011 Clermont-Ferrand, France; ${ }^{2}$ Senvice de Cytogénétique Médicale, Hôpital Gabriel Montpied, 63001 Clermont-Ferrand, France; ${ }^{3}$ INSERM U484, 63005 Clermont-Ferrand, France; ${ }^{4}$ Laboratoire d'Anatomie Pathologique, Hôpital Gabriel Montpied, 63001 Clermont-Ferrand, France; ${ }^{5}$ Senvices de Neurochirurgie, Hôpital Gabriel Montpied, 63001 Clermont-Ferrand, France; ${ }^{6}$ Senvice de Neurochirurgie, Hôpital de la Timone, 13385 Marseille, France; ' ${ }^{3}$ Service Statistiques et Communications Médicales, Centre Jean Perrin, 630 I I Clermont-Ferrand, France

The presence of telomerase activity in a glioma may be a predictor of its malignant potential. Activation of telomerase is regulated at the transcriptional level of the human telomerase reverse transcriptase ( $h T E R T)$. Here, we evaluated whether the amount of hTERT mRNA provides a molecular marker of glioma malignancy that would have clinical utility. We used a real-time RT-PCR to assess the number of hTERT transcripts in primary tumour samples derived from 70 glioma patients. Results were standardised by quantifying the number of $A B L$ transcripts as internal control and expressed as hTERT/ABL ratio. The percentage of patients with detectable hTERT mRNA markedly increased with enhanced malignancy: low-grade gliomas expressed hTERT in one out of 14 cases (7.1\%), anaplastic gliomas in four out of 13 cases (30.8\%) and glioblastoma multiforme (GBM) tumours in 30 out of 43 cases (69.8\%). The mean $h T E R T / A B L$ ratio was significantly higher in GBMs than in non-GBMs. Subdividing hTERT/ABL ratios as low ( $\leqslant 25 \%)$ and high ( $>25 \%$ ), we found that the overall survival among hTERT-positive GBMs was significantly worse in high $h T E R T$ expressors than in low hTERT expressors $(P=0.0082)$. We conclude that the amount of $h T E R T$ mRNA may represent a diagnostic and prognostic indicator for GBM patients.

British Journal of Cancer (2003) 88, 516-520. doi: I0.1038/sj.bjc.6600754 www.bjcancer.com

(c) 2003 Cancer Research UK

Keywords: hTERT; real-time PCR; glioblastoma multiforme; diagnosis; prognosis

Gliomas are the most common of primary brain tumours in humans and form a complex group because of the great variability in degree of malignancy that exists among them. The grade of malignancy is one of the major factors in the prognosis of glioma patients (DeAngelis, 2001). Patients with low-grade tumours typically survive more than 5 years, patients with anaplastic tumours about 3 years, and patients with glioblastoma multiforme (GBM), glioma of the highest grade of malignancy, have the poorest prognosis with median survival of less than 1 year despite modern therapy. Molecular genetic research has led to an increased knowledge of markers that are associated with glioma malignancy and may be useful diagnostic and therapeutic targets. In particular, malignant progression in gliomas has been correlated with the presence of telomerase activity in tumour cells (Langford et al, 1995).

Telomerase is a specialised reverse transcriptase enzyme that maintains telomeres by adding telomeric TTAGGG repeats to the ends of human chromosomes (Morin, 1989). Telomerase is repressed in most normal human somatic cells, and without new synthesis of telomeres the chromosomes shorten with progressive cell division, eventually triggering either replicative senescence or apoptosis when telomere length becomes critically short (Harley

*Correspondence: Dr A Tchirkov, Département de Radiothérapie, Centre Jean Perrin - BP 392, 630 I I Clermont-Ferrand Cedex I, France;

E-mail: andrei.tchirkov@cjp.u-clermont I.fr

Revised 25 September 2002; accepted 19 November 2002 et al, 1990). In contrast, cells that express a sufficient level of telomerase activity escape from replication limitations and are considered as immortal. Reactivation of telomerase is believed to be an essential step during malignant tumour progression (Counter et al, 1994). A strong association between the presence of telomerase and malignancy has been established in nearly all cancer types (reviewed in Shay et al, 2001). Importantly, high telomerase activity is generally associated with high tumour aggressiveness.

Studies of gliomas have shown that more than $50 \%$ of tumours have telomerase activity, detection rates increasing with the grade of malignancy (Langford et al, 1995; Nakatani et al, 1997; Le et al, 1998; Sugita et al, 2000). These observations have suggested that the presence of such activity may indicate the malignant potential of a glioma. However, the prognostic value of telomerase in gliomas remains uncertain. Although telomerase activity has been correlated with inferior survival in low-grade and anaplastic astrocytomas, no clear relation has been determined between the detection or relative level of telomerase activity and patient survival in GBMs, the most common and most malignant gliomas (Nakatani et al, 1997; Hiraga et al, 1998; Huang et al, 1999; Kleinschmidt-Demasters et al, 2000).

The activation of telomerase is tightly regulated at the transcriptional level of the human telomerase reverse transcriptase $(h T E R T)$. Many studies have suggested that the transcription of $h T E R T$ represents the rate-limiting step in telomerase expression, and the detection of $h T E R T$ transcripts using RT - PCR revealed a strong correlation with telomerase activity in the majority of 
tumours (reviewed in Pool et al, 2001). A recent method of realtime PCR has greatly facilitated sensitive and quantitative detection of $h T E R T$ mRNA. In this study, we evaluated the level of $h T E R T$ transcripts in 70 primary gliomas and correlated them with malignancy grade. In GBMs, we also studied the relation between the amount of $h T E R T$ mRNA and patient survival. We found that $h T E R T$ expression increased with malignancy grade and was highly associated with GBMs. Within this important glioma subgroup, the level of $h T E R T$ mRNA was predictive of patient survival.

\section{MATERIALS AND METHODS}

\section{Patients and samples}

Primary tumour samples were obtained from 70 glioma patients at surgical resection of their lesion. The samples for hTERT expression analysis were taken from the material of resection during the course of standard diagnostic procedure. According to the French law on biomedical research, this is an epidemiological study that does not have to be submitted to an Institutional Review Board. Immediately after surgery, the samples were snap-frozen and stored in liquid nitrogen until RNA extraction.

Histological diagnosis and grading of tumours were consistent with the WHO criteria (World Health Organization, 2000). A total of 14 tumours were classified as low-grade gliomas, 13 tumours were anaplastic gliomas, and the remaining 43 tumours were classified as GBMs. The details of tumour type and grade are given in Table 1.

Among 70 glioma patients, 46 patients were male and 24 were female. The mean age at diagnosis was $31.5 \pm 12.2$ years for patients with low-grade tumours, $43.7 \pm 12.7$ years for patients with anaplastic gliomas and $59.5 \pm 10.7$ years for patients with GBMs. The median follow-up was 61 months (range, 32-184 months). In two GBM cases, very early postsurgery death occurred. In most cases, postsurgical treatments included radiotherapy and/or various chemotherapy regimens. Note that in our GBM series, postsurgical treatments had no prognostic impact (data not shown).

\section{Extraction of RNA and preparation of cDNA}

Total RNAs were extracted from tumour samples using Trizol ${ }^{\mathbb{R}}$ reagent (GIBCO/BRL, Grand Island, NY, USA), according to the manufacturer's instructions. Sample RNA $(1 \mu \mathrm{g})$ was reverse transcribed as reported elsewhere (Rolhion et al, 1999). In parallel, reverse transcription reaction was set up without enzyme and then

Table I Detection of hTERT mRNA in gliomas of varying type and malignancy grade

\begin{tabular}{|c|c|c|c|c|}
\hline \multirow[b]{2}{*}{ Type of glioma } & \multirow{2}{*}{$\begin{array}{l}\text { WHO } \\
\text { grade }\end{array}$} & \multirow{2}{*}{$\begin{array}{l}\text { Number } \\
\text { of cases }\end{array}$} & \multicolumn{2}{|c|}{ hTERT MRNA } \\
\hline & & & Positive & Negative \\
\hline Low-grade gliomas & & 14 & I (7.1\%) & $13(92.9 \%)$ \\
\hline Pilocytic astrocytoma & I & 3 & 0 & 3 \\
\hline Diffuse astrocytoma & $\|$ & 2 & 0 & 2 \\
\hline Oligoastrocytoma & $\|$ & 3 & 0 & 3 \\
\hline Oligodendroglioma & $\|$ & 6 & । & 5 \\
\hline Anaplastic gliomas & & 13 & $4(30.8 \%)$ & $9(69.2 \%)$ \\
\hline Anaplastic astrocytoma & III & 2 & 0 & 2 \\
\hline Anaplastic oligoastrocytoma & III & 3 & 0 & 3 \\
\hline Anaplastic oligodendroglioma & III & 8 & 4 & 4 \\
\hline Glioblastoma multiforme & IV & 43 & $30(69.8 \%)$ & $13(30.2 \%)$ \\
\hline
\end{tabular}

the sample was processed to PCR as a control for DNA contamination.

\section{Quantitative real-time RT - PCR for hTERT mRNA}

We assessed the number of $h T E R T$ mRNA transcripts using realtime PCR in the LightCycler system (Roche Diagnostics, Meylan, France). The amplification of hTERT mRNA was performed with the DNA Master SYBRGreen I reagent set (Roche Diagnostics, Meylan, France) using the following primers: forward primer $5^{\prime}$ GGAGCAAGTTGCAAAGCATTG- $3^{\prime}$ and reverse primer $5^{\prime}$ TCCCACGACGTAGTCCATGTT- $3^{\prime}$. Quantification of $A B L$ transcripts as an internal control for the amount and quality of cDNA was performed for all samples, using the forward primer $5^{\prime}$ GCCGCTCGTTGGAACTCCAAGG- $3^{\prime}$ and reverse primer $5^{\prime}$ TGACTGGCGTGATGTAGTTTGCTT-3' (Tchirkov et al, 1998). Several studies have confirmed that the $A B L$ gene is an appropriate reference in quantitative real-time PCR studies of both pathological and normal cells (Buonamici et al, 2002; Moniotte et al, 2001). During initial optimisation of PCR conditions, amplified products were analysed using agarose gels to ensure correct product size. Once the PCR product size was verified, the melting temperature of the product determined with LightCycler meltingcurve analysis was used to control for the specificity of amplifications. The number of transcripts in samples was calculated with the LightCycler software, using the calibration data obtained with serial dilutions of purified PCR products containing known numbers of cDNA molecules of each gene (Figure 1). The results of real-time PCR were given as the ratio between $h T E R T$ and $A B L$ transcripts, expressed as a percentage. Glioma samples were analysed in a blind-trial fashion. All experiments were performed in triplicate, with good consistency of results (the mean coefficient of variation was $8.4 \%$ ).

\section{Statistical analysis}

The overall survival time was calculated in months after initial surgery date. For patients with $h T E R T$-expressing GBMs, a receiver operating characteristic (ROC) analysis was used to determine which $h T E R T$ level best discriminated between those patients who did not reach the median survival time (15 months for hTERTpositive GBM patients) and the others (Bamber, 1975; Hanley and McNeil, 1982). This method was valid since all patients had died by the time of analysis. Kaplan-Meier estimation was used to evaluate the overall survival and the log-rank statistics was used to compare survival between subgroups of patients (Kaplan and Meier, 1958). Other analyses of statistical links between biological and clinical parameters were performed using standard tests.

\section{RESULTS}

\section{hTERT mRNA expression in gliomas}

We studied 70 gliomas for the presence of $h T E R T$ mRNA using a real-time RT-PCR. Table 1 shows that low-grade gliomas expressed $h T E R T$ in one out of 14 cases $(7.1 \%)$, anaplastic gliomas in four out of 13 cases (30.8\%) and GBMs in 30 out of 43 cases (69.8\%). Thus, the frequency of hTERT expression significantly increased with advanced glioma malignancy $\left(\chi^{2}\right.$-test, $\left.P=0.00024\right)$. Moreover, on comparing GBMs and non-GBMs, we found that the expression of $h T E R T$ was highly associated with GBMs $\left(\chi^{2}\right.$-test, $P=0.00003)$. Among non-GBMs, the expression of $h T E R T$ was detected mainly in anaplastic oligodendrogliomas.

In $h T E R T$-positive glioma samples, the number of $h T E R T$ transcripts was assessed and normalised to the expression of a housekeeping gene, $A B L$. Among glioma patients with detectable hTERT MRNA, the hTERT/ABL ratio ranged between 2.6 and $180.1 \%$ (Figure 2). The $h T E R T / A B L$ ratio was significantly different 

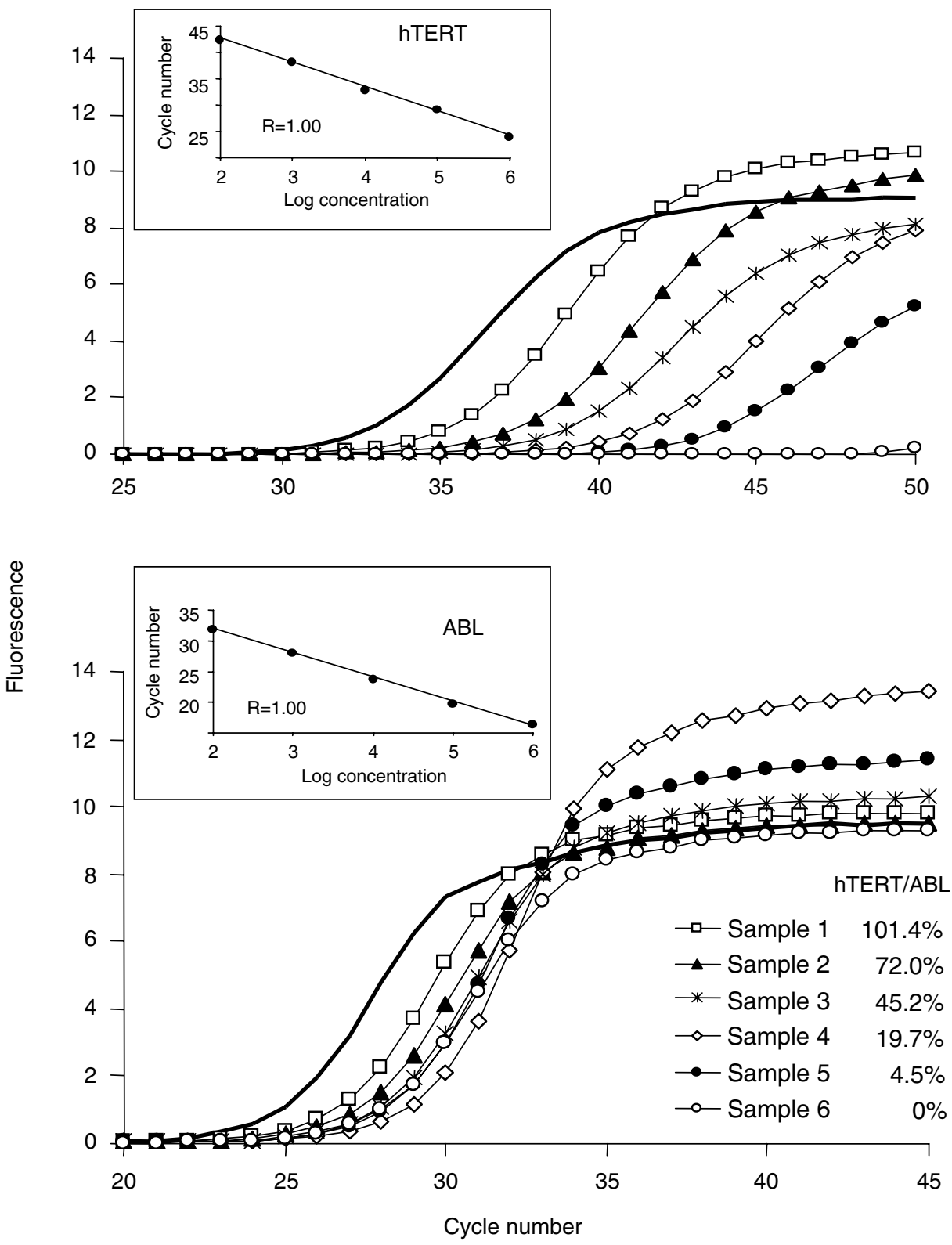

Figure I Example of real-time PCR quantification of $h T E R T$ in tumour samples. Amplification profiles obtained on the LightCycler are presented for $h T E R T$ (upper graph) and $A B L$ (lower graph) transcripts. Linear regressions of standard dilution series, indicating accuracy of the analysis, are shown on the upper left part of each graph. Calculation of the number of $h T E R T$ and $A B L$ transcripts was done by using these standard curves. One of the standards was included in each PCR run (amplification profiles shown as solid lines, $10^{5}$ molecules). For each sample, the number of $h T E R T$ transcripts was divided by the number of $A B L$ transcripts in order to standardise the level of hTERT mRNA. The obtained ratios expressed as percentage are shown.

between the low-grade, anaplastic and GBM tumour groups (Kruskal-Wallis test, $P=0.00013$ ). Among patients with detectable levels of $h T E R T$ mRNA, the amount of $h T E R T$ transcripts was significantly higher among the GBM patients $(54.2 \%$, mean) than in non-GBMs ( $10.2 \%$, mean) (Mann-Whitney $U$-test, $P=0.012)$. Overall, the level of $h T E R T$ expression was neither age- (Spearman rank test, NS) nor gender-related (Kruskal-Wallis test, NS).

\section{Prognostic significance of hTERT expression in GBMs}

In the GBM group, we tested for a possible relation between the relative level of $h T E R T$ expression and patient survival. The cases with gross total tumour excision $(n=5)$, where no residual contrast enhancement was detected on early postoperative computed tomography scans, were excluded from analysis, as complete resection of the lesion could influence favourably the survival in GBMs (Scott et al, 1999). Among the 36 patients with subtotal resection of their lesion, 25 had $h T E R T$ positive tumours. In the $h T E R T$-positive patient group, we determined, using an ROC analysis, a cut-off level of $h T E R T$ transcripts of $23.7 \%$ that best segregated patients into poor- and good-prognosis subgroups (as described in the Statistical Analysis). We found that the patients with high (>25\%) hTERT mRNA levels had significantly shorter survival $v s$ the patients with low $(\leqslant 25 \%)$ levels (log-rank test, $P=0.0082$, Figure 3 ). The 11 patients with non-detectable hTERT mRNA had survival equivalent to that of high $h T E R T$ expressors (log-rank test, $P=0.91$, NS). The survival of these patients was also significantly worse than that of patients with low hTERT levels (log-rank test, $P=0.0064$ ). 


\section{DISCUSSION}

In this study, we measured the hTERT mRNA level in tumour specimens from a cohort of 70 patients with gliomas using a recently introduced real-time quantitative PCR technique. We found a progressive increase in the $h T E R T$ detection rate with increasing grade of glioma malignancy: $7 \%$ for low-grade gliomas,

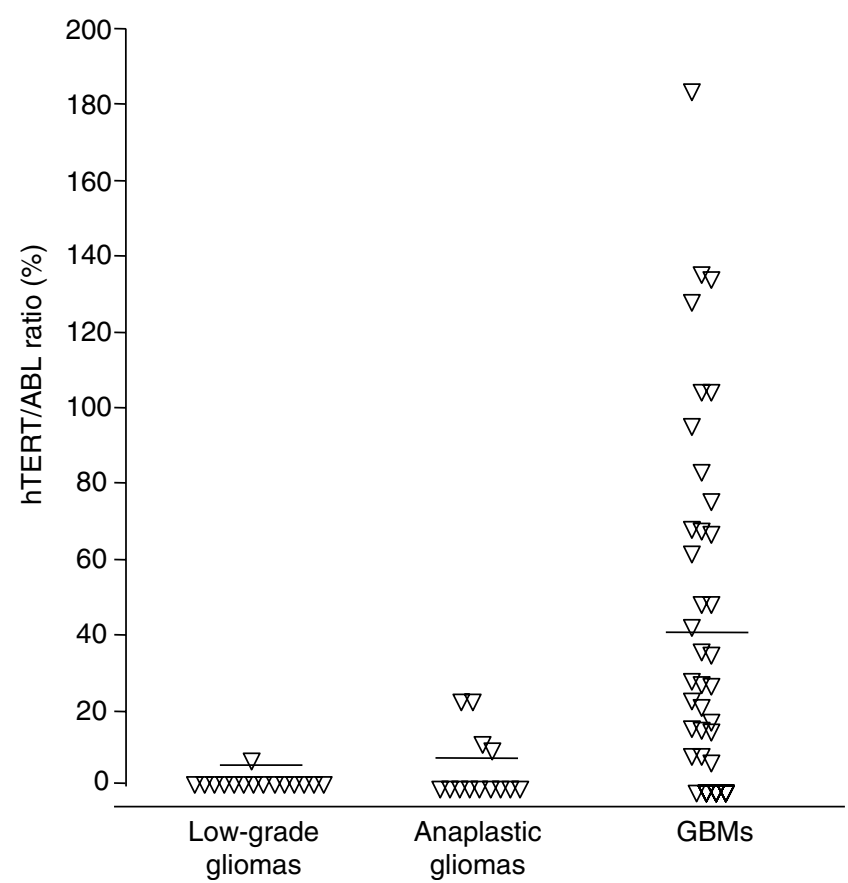

Figure 2 Individual $h T E R T / A B L$ ratios (\%) determined in glioma samples according to the investigated tumour types. The mean values are shown as horizontal bars for each group: low-grade tumours (0.2\%), anaplastic gliomas (3.7\%), GBMs (37.8\%).
$31 \%$ for anaplastic gliomas and $70 \%$ for GBMs. These data are in agreement with the detection rates of telomerase activity reported for these tumour types (Hiraga et al, 1998; Le et al, 1998; Harada et al, 2000; Sugita et al, 2000). The association of hTERT expression with GBMs was highly specific when comparing GBMs to non-GBMs. This observation is in line with the recent demonstration of a similar association at the level of $h T E R T$ protein overexpression (Chakravarti et al, 2001). Among non-GBMs, $h T E R T$ mRNA was detectable in anaplastic oligodendrogliomas, consistent with a previous report (Langford et al, 1995). Among patients with detectable levels of $h T E R T$ mRNA, the amount of $h T E R T$ transcripts was significantly higher among the GBM patients. Taken together, these findings suggest that the level of $h T E R T$ mRNA may be used as an indicator of enhanced glioma malignancy. The hTERT analysis may complement histology and help to refine tumour grading and classification.

Current system of pathological grading for human gliomas is often nonprognostic: some tumours responding well to treatment may be histologically indistinguishable from nonresponding ones. Previous studies have suggested that telomerase activity in gliomas may have utility in tumour prognosis, as the presence of such activity has been correlated with a poor prognosis for low-grade and anaplastic tumours (Nakatani et al, 1997; Hiraga et al, 1998; Huang et al, 1999). However, studies have failed to identify any significant relation between the level of telomerase activity and patient survival in GBMs (Nakatani et al, 1997; Hiraga et al, 1998; Kleinschmidt-Demasters et al, 2000). In the present study, we found that among $h T E R T$-positive GBM patients, the overall survival was significantly worse in the patients with high levels of $h T E R T$ mRNA. This finding is compatible with the result regarding hTERT-protein overexpression in GBMs, which showed that high levels of the protein correlated with reduced patient survival (Chakravarti et al, 2001). Importantly, the patients without detectable $h T E R T$ mRNA had a short survival equivalent to that of high $h T E R T$ expressors. In contrast, the patients with low levels of $h T E R T$ had prolonged survival. Thus, the level of $h T E R T$ mRNA may predict decreased or increased survival in GBMs.

The level of $h T E R T$ mRNA estimated with real-time RT-PCR procedure is the average amount of transcripts in a whole tumour

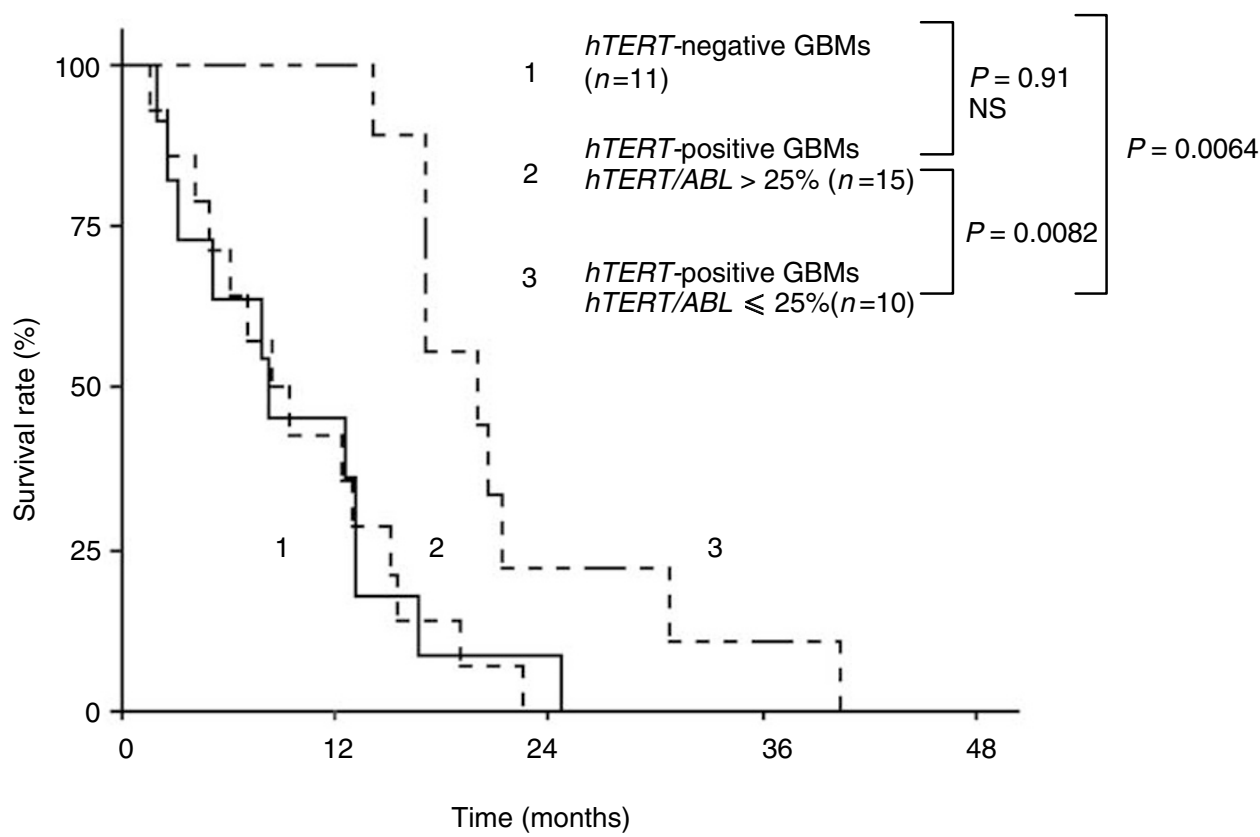

Figure 3 Survival of GBM patients in relation to hTERT status in their tumours. Patients, whose tumours expressed hTERT at low levels, survived significantly longer (19 months, median) than those patients who showed high hTERT levels in their tumours ( 8 months, median) or than hTERT-negative patients ( 8 months, median) did. Note the very similar survival period for high hTERT expressors and hTERT-negative patients. 
sample and mainly depends on the number of $h T E R T$-positive cells present in the tumour tissue. Studies of $h T E R T$-protein distribution using immunohistochemistry in cultured cells and tissue sections have shown that $h T E R T$ expression was detected in almost all neoplastic cells in cancer tissues with high telomerase activity, whereas cancers with low telomerase activity had fewer $h T E R T$ positive cancer cells (Hiyama et al, 2001). The burden of $h T E R T$ positive cells probably reflects the degree of expansion of clonogenic tumour cell population, which has a great selective advantage and proliferation capacity provided by telomerase activity. It is possible that the level of expansion correlates with the size of clonogenic cell fraction. Thus, low $h T E R T$ expressors might survive longer than high $h T E R T$ expressors since they are likely to have fewer neoplastic stem cells at the time of diagnosis, and cytotoxic treatments are in this case more efficient. Nevertheless, testing this hypothesis requires further studies of $h T E R T$ positive gliomas combining the evaluation of $h T E R T$-protein expression at the individual cell level and clonogenic assays.

Equally poor prognosis found for GBM patients in the groups with high $h T E R T$ expression and without $h T E R T$ expression points to the fact that aggressive growth of some GBMs may occur in the absence of telomerase. Telomerase-negative GBMs might achieve immortalisation by an alternative mechanism of telomere length stabilisation. Evidence in support of this hypothesis has been reported in a study of telomere length in gliomas (Morii et al, 1997). Compared with telomerase-positive gliomas, telomerasenegative gliomas were found to have very long and heterogeneous telomeres, characteristics seen in tumour cells that have acquired an alternative mechanism for lengthening their telomeres. The nature of this mechanism named alternative lengthening of telomeres (ALT) and described in several tumour types is currently unclear, but may involve nonreciprocal recombination between telomeres (Bryan et al, 1997). Given the poor prognosis in $h T E R T$ negative patients, it seems important to investigate the possible role of the ALT mechanism in malignant progression of GBMs.

In conclusion, hTERT mRNA expression may be used as a molecular marker of glioma malignancy that may be particularly helpful in diagnosing GBMs as a complement to existing approaches. In addition, the level of $h T E R T$ transcripts appears to predict in GBMs decreased or increased survival. In the development and future application of anti-telomerase treatments of malignant gliomas (Komata et al, 2002), hTERT analysis of any given tumour will be essential.

\section{REFERENCES}

Bamber D (1975) The area above the ordinal dominance graph and the area below the receiver operating characteristic graph. J Math Psychol 12: $387-395$

Bryan TM, Reddel RR (1997) Telomere dynamics and telomerase activity in in vitro immortalised human cells. Eur J Cancer 33: 767-773

Buonamici S, Ottaviani E, Testoni N, Montefusco V, Visani G, Bonifazi F, Amabile M, Terragna C, Ruggeri D, Piccaluga PP, Isidori A, Malagola M, Baccarani M, Tura S, Martinelli G (2002) Real-time quantitation of minimal residual disease in inv(16)-positive acute myeloid leukemia may indicate risk for clinical relapse and may identify patients in a curable state. Blood 99: $443-449$

Chakravarti A, Delaney MA, Noll E, Black PM, Loeffler JS, Muzikansky A, Dyson NJ (2001) Prognostic and pathologic significance of quantitative protein expression profiling in human gliomas. Clin Cancer Res 7: 2387 2395

Counter CM, Hirte HW, Bacchetti S, Harley CB (1994) Telomerase activity in human ovarian carcinoma. Proc Natl Acad Sci USA 9: 2900-2904

DeAngelis LM (2001) Brain tumors. N Engl J Med 344: 114-123

Hanley JA, McNeil BJ (1982) The meaning and use of the area under a receiver operating characteristic (ROC) curve. Radiology 143: 29-36

Harada K, Kurisu K, Tahara H, Tahara E, Ide T, Tahara E (2000) Telomerase activity in primary and secondary glioblastomas multiforme as a novel molecular tumor marker. J Neurosurg 93: 618-625

Harley CB, Futcher AB, Greider CW (1990) Telomeres shorten during ageing of human fibroblasts. Nature 345: $458-460$

Hiraga S, Ohnishi T, Izumoto S, Miyahara E, Kanemura Y, Matsumura H, Arita N (1998) Telomerase activity and alterations in telomere length in human brain tumors. Cancer Res 58: 2117-2125

Hiyama E, Hiyama K, Yokoyama T, Shay JW (2001) Immunohistochemical detection of telomerase ( $h T E R T)$ protein in human cancer tissues and a subset of cells in normal tissues. Neoplasia 3: 17-26

Huang F, Kanno H, Yamamoto I, Lin Y, Kubota Y (1999) Correlation of clinical features and telomerase activity in human gliomas. J Neuro-oncol 43: $137-142$

Kaplan E, Meier P (1958) Nonparametric estimation from incomplete observations. J Am Stat Assoc 53: $457-481$

Kleinschmidt-Demasters BK, Evans LC, Bobak JB, Lopez-Uribe D, Hopper D, Shroyer AL, Shroyer KR (2000) Quantitative telomerase expression in glioblastomas shows regional variation and down-regulation with therapy but no correlation with patient outcome. Hum Pathol 31: 905-913

Komata T, Kanzawa T, Kondo Y, Kondo S (2002) Telomerase as a therapeutic target for malignant gliomas. Oncogene 21: 656-663
Langford LA, Piatyszek MA, Xu R, Schold SC, Shay JW (1995) Telomerase activity in human brain tumours. Lancet 346: 1267-1268

Le S, Zhu JJ, Anthony DC, Greider CW, Black PM (1998) Telomerase activity in human gliomas. Neurosurgery 42: 1120-1124

Moniotte S, Vaerman JL, Kockx MM, Larrouy D, Langin D, Noirhomme P Balligand JL (2001) Real-time RT-PCR for the detection of betaadrenoceptor messenger RNAs in small human endomyocardial biopsies. J Mol Cell Cardiol 33: $2121-2133$

Morii K, Tanaka R, Onda K, Tsumanuma I, Yoshimura J (1997) Expression of telomerase RNA, telomerase activity, and telomere length in human gliomas. Biochem Biophys Res Commun 239: 830-834

Morin GB (1989) The human telomere terminal transferase enzyme is a ribonucleoprotein that synthesizes TTAGGG repeats. Cell 59: $521-529$

Nakatani K, Yoshimi N, Mori H, Yoshimura S, Sakai H, Shinoda J, Sakai N (1997) The significant role of telomerase activity in human brain tumors. Cancer 80: $471-476$

Pool JC, Andrews LG, Tollefsbol TO (2001) Activity, function, and gene regulation of the catalytic subunit of telomerase $(h T E R T)$. Gene 269: $1-12$

Rolhion C, Penault-Llorca F, Chevillard S, Verrelle P, Finat-Duclos F (1999) Quantification of RT - PCR products: assessment of ethidium bromide stained gel analysis in comparison with fluorescent detection using an automated sequencer. Lab Med 30: 419-422

Scott JN, Rewcastle NB, Brasher PM, Fulton D, MacKinnon JA, Hamilton M, Cairncross JG, Forsyth P (1999) Which glioblastoma multiforme patient will become a long-term survivor? A population-based study. Ann Neurol 46: $183-188$

Shay JW, Zou Y, Hiyama E, Wright WE (2001) Telomerase and cancer. Hum Mol Genet 10: 677-685

Sugita Y, Nakashima A, Kato S, Sakata K, Morimatsu M, Shigemori M (2000) Telomerase activity in gliomas with the use of non-radioisotopic and semi-quantitative procedure for terminal repeat amplification protocol. Oncol Rep 7: 1087-1092

Tchirkov A, Giollant M, Tavernier F, Briançon G, Tournilhac O, Kwiatkowski F, Philippe P, Choufi B, Deméocq F, Travade P, Malet P (1998) Interphase cytogenetics and competitive RT-PCR for residual disease monitoring in patients with chronic myeloid leukemia during interferon- $\alpha$ therapy. Br J Haematol 101: $552-557$

World Health Organization (2000) WHO Classification Astrocytic tumors. Oligodendroglial tumours and mixed gliomas. In Pathology and Genetics of Tumours of the Nervous System, Kleihues P, Cavenee WK (eds) pp 6-70. Lyon: IARC Press 